ACCEPted For Publication in ApJ LetTers

Preprint typeset using LATEX style emulateapj v. 08/22/09

\title{
HUBBLE SPACE TELESCOPE IMAGES OF RED MERGERS: HOW DRY ARE THEY?
}

\author{
KATHerine E. Whitaker ${ }^{1}$ \& Pieter G. VAN DoKkum ${ }^{1}$ \\ Accepted for publication in ApJ Letters
}

\begin{abstract}
Mergers between red galaxies are observed to be common in the nearby Universe, and are thought to be the dominant mechanism by which massive galaxies grow their mass at late times. These "dry" mergers can be readily identified in very deep ground based images, thanks to their extended low surface brightness tidal features. However, ground-based images lack the required resolution to determine the morphologies of the merging galaxies, and to measure the amount of dust and associated gas. We present HST/ACS and WFPC2 observations of a sample of 31 bulge-dominated red-sequence galaxies at $z \sim 0.1$, comprised of ongoing mergers, merger remnants, and undisturbed galaxies. Nearly all galaxies have early-type morphologies and most are well-fit by $\mathrm{r}^{1 / 4}$ law surface brightness profiles. We find that only $10 \%$ of the galaxies show evidence for the presence of dust. The amount of cold gas (or its upper limit) is calculated from the mean color-excess, assuming a simple relation between gas mass and dust mass. The gas mass is low for all galaxies, and we find that $\mathrm{M}_{\text {gas }} / \mathrm{M}_{\text {stellar }} \lesssim 3 \times 10^{-4}$. We infer that red mergers in the nearby Universe mostly involve early-type galaxies containing little cold gas and dust. This may imply that the progenitors were mostly devoid of gas and/or that feedback mechanisms are very effective in preventing the gas to cool. The lack of gas in these objects may also imply a relatively large fraction of binary black holes in the centers of massive ellipticals.
\end{abstract}

Subject headings: galaxies: elliptical - galaxies: evolution - galaxies: formation

\section{INTRODUCTION}

In currently popular models massive elliptical galaxies are expected to have assembled a significant fraction of their final mass at redshifts $z<1$ through mergers (e.g., de Lucia et al. 2006). As elliptical galaxies follow tight scaling relations and have old stellar populations, these mergers cannot involve large amounts of gas and associated star formation. Instead, recent studies have advanced the idea of the continued assembly of elliptical galaxies through mergers between galaxies which are already on the red sequence (e.g., van Dokkum et al. 1999, Bell et al. 2004, 2006, van Dokkum 2005 [hereafter, vD05], Tran et al. 2005, White et al. 2007, Faber et al. 2007, Zheng et al. 2007, McIntosh et al. 2007). These so-called "dry" mergers are now thought to be the dominant mode of growth of massive $\left(>\right.$ several $M_{\star}$ ) galaxies at $0<z<1$, building up the high-mass end of the mass function but not changing the overall mass density of ellipticals.

It is not yet clear how much gas is involved in these mergers, that is, how dry they are. The amount of gas provides an important constraint on the efficiency of feedback in galaxy formation models (see, e.g., Croton et al. 2006), and may also determine the fate of the supermassive black holes of the progenitor galaxies (e.g., Escala et al. 2004). The red colors of massive mergers suggest that their light is dominated by old stellar populations, but may also reflect the presence of dust. Furthermore, we have only very limited information on the morphologies of galaxies involved in dry mergers. Recent simulations by Feldmann et al. (2008) have demonstrated that some

\footnotetext{
Electronic address: katherine.whitaker@yale.edu

${ }^{1}$ Department of Astronomy, Yale University, P.O. Box 208101, New Haven, CT 06520
}

tidal features observed by vD05 are not necessarily the result of spheroid-spheroid mergers, but could also be kinematically cold material from the merger of a spheroid and a disk-dominated system.

Deep ground-based images are excellent for detecting low surface-brightness features, but their limited resolution makes it difficult to address these questions. The ongoing mergers and merger remnants identified in vD05 have redshifts of $z \sim 0.1$, and are barely resolved within the effective radius (assuming $r_{e} \approx 3 \mathrm{kpc}$ and $\approx 1^{\prime \prime}$ seeing). Here we present Hubble Space Telescope (HST) images with the Advanced Camera for Surveys (ACS) and the Wide Field and Planetary Camera 2 (WFPC2) for a representative subset of galaxies drawn from the vD05 sample of luminous red galaxies. The resolution offered by HST allows us to determine the morphologies of the red mergers and their remnants, and to determine the amount of dust associated with these mergers.

\section{DATA AND REDUCTION}

The sample consists of 31 galaxies, selected out of a sample of 86 bright bulge-dominated, red galaxies at a redshift of $\mathrm{z} \sim 0.1$ discussed by $\mathrm{vD} 05$. According to the visual tidal classification in vD05, the sample contains 3 undisturbed galaxies, 5 weakly disturbed remnants, 5 strongly disturbed remnants, and 9 ongoing merging pairs. Each target was observed for one orbit, split in two exposures in the $V_{606}$ filter and two exposures in the $I_{814}$ filters. Fourteen galaxies were observed with ACS, using the Wide Field Channel (WFC). The instrument stopped operating due to an electrical short in January 2007 , so the remaining 17 galaxies were observed with the Wide Field and Planetary Camera 2 (WFPC2).

The targets observed by ACS have two $512 \mathrm{~s}$ dithered exposures in each filter, enabling cosmic-ray detection. 

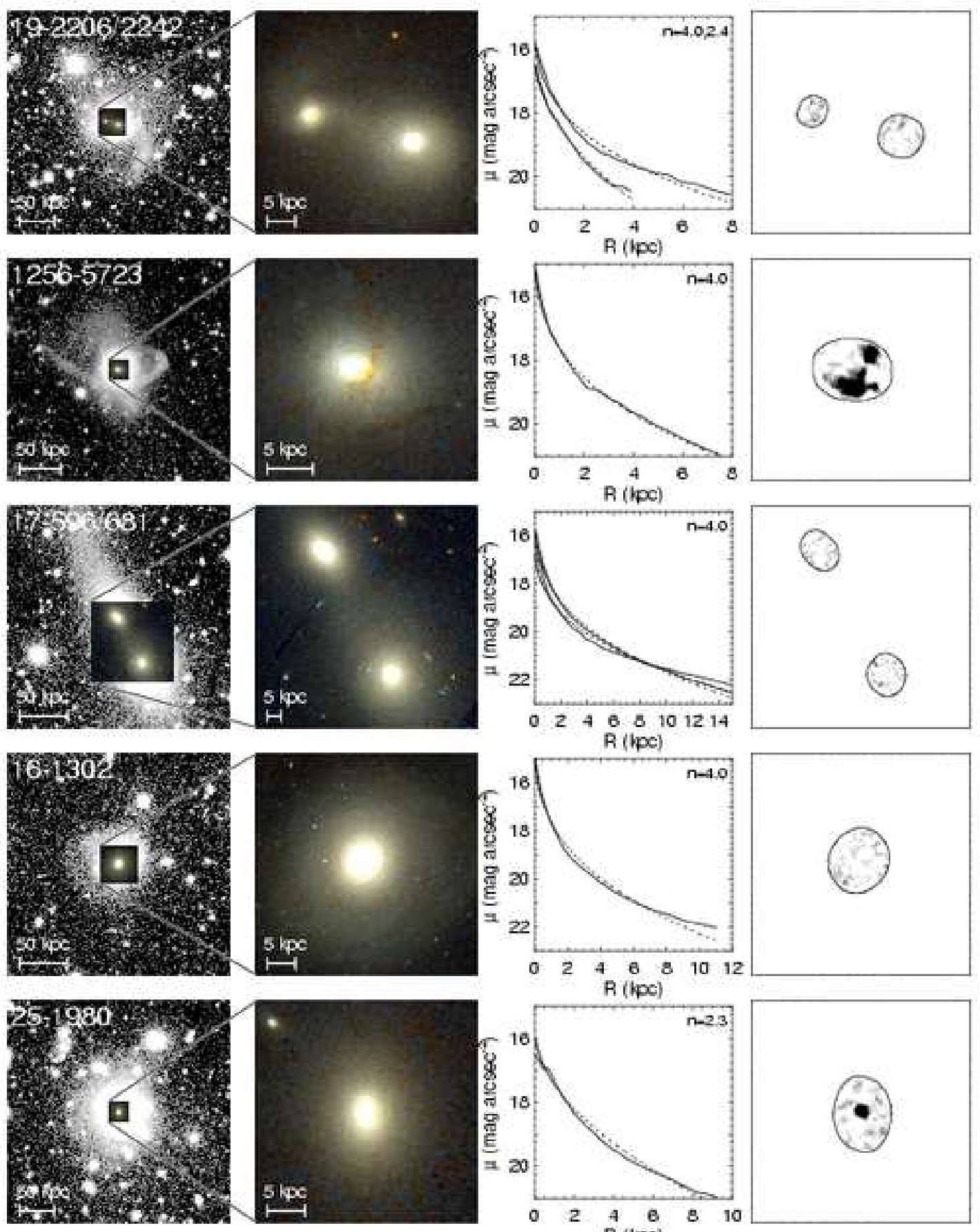

FIG. 1. - Seven examples of galaxies from our sample: two ongoing mergers and three objects with strong or weak tidal features (from vD05). The left panels show very deep ground-based images used to identify the tidal features in vD05. The second column shows color images generated from the $V_{606}$ and $I_{814}$ HST data. Also shown are surface brightness profiles (solid lines) with the best-fit Sersic profiles overplotted (broken lines), and color excess images, where black implies an excess of red light (i.e., dust).

The ACS calibration pipeline performs the basic image reduction and removal of cosmic-rays, as well as removing the geometric distortions and combining the dithered data. The amplifiers on either side of the chip resulted in two different background values in the pipeline product. This artifact was removed by subtracting the median background value for each amplifier.

The targets observed by WFPC 2 have two $400 \mathrm{~s}$ dithered exposures. The IRAF, STSDAS tasks warmpix and crrej remove warm pixels and cosmic-rays to improve the standard pipeline calibrated data. The task L.A.Cosmic (van Dokkum 2001) is further used to improve the cosmic-ray removal.

\section{MORPHOLOGIES}

The majority of the sample appear to be normal earlytype galaxies with no visible dust signatures. The galaxies generally have smooth surface brightness profiles with 
little isophotal twisting or changes in ellipticity. Low surface-brightness tidal features are present in the ongoing mergers and disturbed galaxies, but not as prominent as in the deeper ground-based images presented in vD05. Fig. 1 contains examples of galaxies in the sample with both ground-based imaging and two-color HST images. Galaxies with striking tidal features in the ground-based data (e.g., 19-2206/2242 and 17-596/681) appear regular in the shallow, high-resolution HST images.

To quantify the properties of the galaxies, we fit the Sersic (1968) $\mathrm{r}^{1 / n}$ law to the surface brightness profiles. The free parameter $n$ is a quantitative measure of the bulge-to-disk ratio: late-type spirals typically are dominated by exponential disks and have $n \sim 1$, elliptical galaxies usually have $n \sim 4$, and galaxies with a combination of varying sized bulges and disks cover the middle ground. Figure 2 shows the distribution of best-fitting Sersic parameters. Most galaxies are well described with de Vaucouleurs profiles with $n \sim 4$, but not all. Different types of shading indicate differences in the presence and extent of tidal features; there is no correlation between the tidal features and the Sersic parameter.

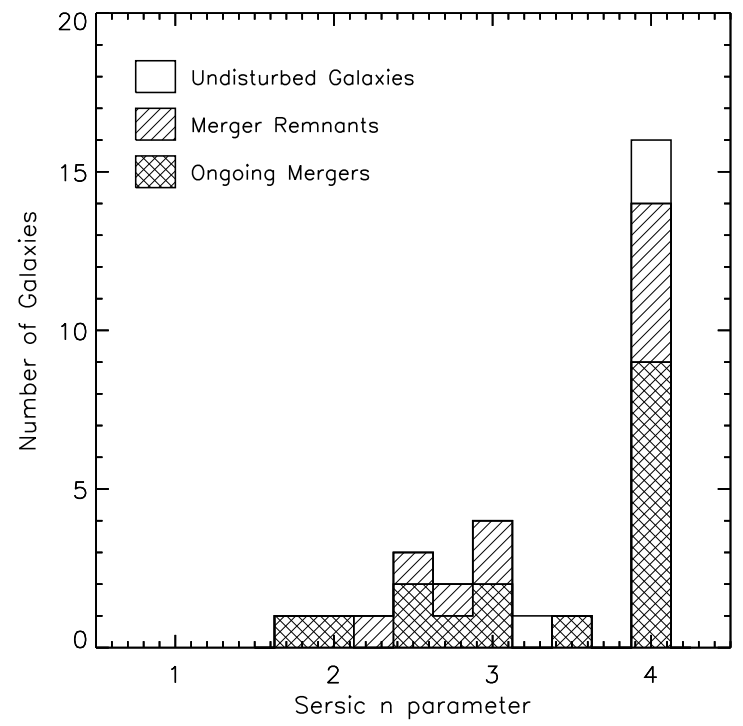

FIG. 2.- The distribution of the minimum $\chi^{2}$ best-fit Sersic $n$ parameter, for different visual tidal classifications. Undisturbed galaxies are open histograms, merger remnants are diagonally hashed lines, and ongoing mergers are cross hashed lines. Most galaxies appear to be bulge-dominated, and there is no correlation between the extent of tidal features and the best-fitting Sersic index.

\section{DERIVING THE GAS MASS}

The majority of the galaxies look like normal earlytype galaxies, but a few show small dips and other deviations in their surface brightness profiles (e.g., 1256-5723 and 25-1980 in Fig. 1). As the dips are more pronounced in the $V_{606}$ band than in the $I_{814}$ band, the cause is likely to be obscuration by dust. In the case of 1256-5723, this is confirmed by the visual appearance of the galaxy: a prominent dust lane winds across the galaxy in the HST image. Assuming that reddening traces dust, the dust mass can be constrained from the color-excess. The color excess images are constructed as follows:

$$
\begin{aligned}
E\left(V_{606}-I_{814}\right) & =\left(V_{606}-I_{814}\right)-\left(V_{606}-I_{814}\right)_{\text {model }} \\
& =-2.5 \log \left(\frac{\left[i\left(V_{606}\right) / i\left(I_{814}\right)\right]}{\left[i\left(V_{606}\right) / i\left(I_{814}\right)\right]_{\text {model }}}\right)
\end{aligned}
$$

where $i\left(V_{606}\right)$ and $i\left(I_{814}\right)$ are intensities and $V_{606}$ and $I_{814}$ are magnitudes. The model $V_{606}-I_{814}$ color image contains no reddening due to dust, and is generated using an ellipse fit with the visible dust regions masked. The color excess images are found in the last column in Fig. 1. with darker regions signifying the presence of dust.

The reddening can be related to the mass in cold gas, assuming that the dust traces cold gas, a mass ratio of $\mathrm{H}$ to $\mathrm{He}$ of 3:1, and an $\mathrm{H}$ gas-to-dust mass ratio of 100 (see, e.g., van Dokkum \& Franx 1995; Tomita et al. 2000):

$$
M_{\text {gas }}\left[M_{\odot}\right]=\Sigma\left\langle E_{V_{606}-I_{814}}\right\rangle \Gamma_{V_{606}-I_{814}},
$$

where $\Sigma$ is the area of the feature $\left(\mathrm{kpc}^{2}\right),\left\langle E_{V-I}\right\rangle$ is the mean reddening in that area, and $\Gamma_{V-I}$ is the mass reddening coefficient . We adopt $\Gamma_{V-I} \sim 5.6 \times 10^{7} \quad \mathrm{M}_{\odot} \mathrm{mag}^{-1} \mathrm{kpc}^{-2}$, which was derived from the Galactic mass absorption coefficient in the $V$ band (Sadler \& Gerhard 1985) and assuming that $E\left(V_{606}-I_{814}\right)_{z=0.1}=0.38 A_{V, z=0}$ (Rieke \& Lebofsky 1985; Cardelli et al. 1989). The mean color excess value is measured only within the area that is $>5 \sigma$ above the background noise (shown as a black contour in the color excess images of Fig. 1).

Only 3 out of the 31 red galaxies $(10 \%)$ have significant detectable dust, with cold gas mass values ranging from 7.7-7.9 in $\log \mathrm{M}_{\odot}$. Of these 3 galaxies, one is an Sa galaxy with a striking dust lane that was probably misclassified in vD05. The other two galaxies are shown in Fig. 1 (1256-5723 and 25-1980). The detection of dust in 12565723 is probably related to the startling tidal arm seen in ground-based images (see vD05, Feldmann et al. 2008). This object may be an isolated example of a merger with a galaxy with a prominent cold component.

For the remaining 28 galaxies $(90 \%)$ we find no evidence for dust. Upper limits on the dust masses in these galaxies were derived from the median absolute deviation of the distribution of mean color excess values. To put the cold gas mass values of all 31 massive red galaxies in context, we determine the gas-to-stellar mass ratio, using stellar mass values from catalogues by Kauffmann et al. (2003) and Gallazi et al. (2005). For those 11 galaxies missing from both catalogues, the stellar masses were estimated from the empirical relationship between $R$-band magnitude (from vD05) and stellar mass defined by the rest of the galaxies. The uncertainty in the masses of these galaxies is $\sim 0.1 \mathrm{dex}$, smaller than the uncertainty in the gas masses.

Figure 3 shows the ratio of cold gas to stellar mass as a function of the quantitative tidal parameter $t$ defined in vD05. This parameter describes the level of distortion by measuring the median absolute deviation of the residuals from model fits. The gas mass in these galaxies is remarkable small, amounting to less than $0.1 \%$ of the stellar mass even for the galaxies where dust was detected. 


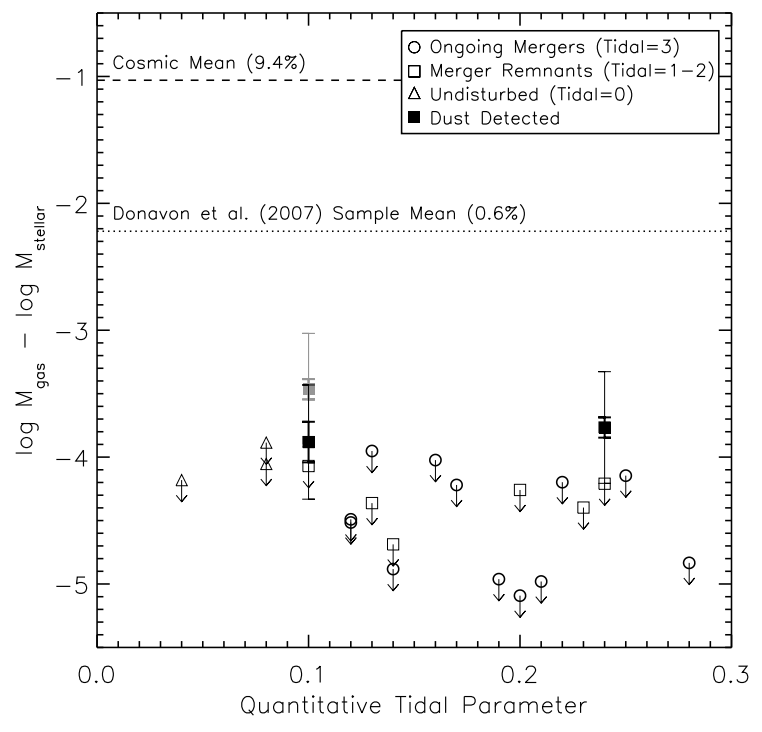

FIG. 3. - Cold gas to stellar mass ratio in red galaxies with varying levels of tidal distortions (quantitative classification from vD05). All galaxies have $\mathrm{M}_{\text {gas }} / \mathrm{M}_{\text {stellar }} \lesssim 3 \times 10^{-4}$. Downward pointing arrows indicate upper limits of the cold gas mass. Those galaxies with significant dust detections are solid points (with the misclassified Sa galaxy in gray), including random and systematic (larger) error bars. The cosmic mean (dashed line) and the mean of the Donavon et al. sample (dotted line) are included for reference.

\section{DISCUSSION}

In this work, we use high-resolution images taken with HST of red mergers identified in vD05 to determine the morphologies of red mergers and to measure the amount of dust and associated cold gas. Nearly all galaxies have early-type morphologies and most are well-fit by $\mathrm{r}^{1 / 4}$ law surface brightness profiles. We find that 90\% of the galaxies exhibit no visible dust signatures, while $10 \%$ have detectable dust. The gas-to-stellar mass ratio is low for all galaxies, including the three with detected dust, where $\mathrm{M}_{\text {gas }} / \mathrm{M}_{\text {stellar }} \lesssim 3 \times 10^{-4}$.

It is interesting to compare this result to those of Donovan et al. (2007), who examined a sample of red elliptical galaxies with significant amounts of neutral hydrogen (as traced by $21 \mathrm{~cm}$ observations). The mean gas-to-stellar mass ratio of the Donovan et al. galaxies that would have been selected by the vD05 criterion is 0.006 , about an order of magnitude greater than the red ellipticals in this

2 Note that almost all elliptical galaxies at $z \sim 0$ have trace amounts of gas and dust at levels substantially below our detection study. This implies that the HI-rich systems studied by Donovan et al. are not representative for the general population of red mergers, that the dust is not a good tracer of the cold gas in these systems, or that the cold gas typically resides at large radii $\left(\gg 1 r_{e}\right)$ and hence would escape detection in our study.

The most straightforward interpretation of our results is that red mergers in the nearby Universe mostly involve early-type galaxies containing very little cold gas. Elliptical galaxies generally have substantial amounts of hot gas and it appears that this gas is not able to condense and form stars, even during mergers. Central active nuclei may play a role in preventing the gas from cooling (e.g., Croton et al. 2006), and it will be interesting to determine whether the merger remnants show enhanced low-level AGN activity.

The absense of large amounts of gas in these objects ${ }^{2}$ has interesting implications for the growth of supermassive black holes. It has been argued that the massive gas disks observed in the centers of gas-rich mergers may be instrumental in removing angular momentum from the binary black holes that result from a major merger (e.g., Escala et al. 2005). As pointed out by Hoffman \& Loeb (2007), dry mergers of massive galaxies with little gas should lead to many stable black hole binaries, and subsequently to unstable triples. The lightest of the three black holes can then be ejected, which has interesting consequences. Our data lend indirect support to such scenarios. A further possible implication is that the black hole merger rate, which is relevant for gravitational wave detection experiments, does not necessarily track the galaxy merger rate.

One of the main uncertainties in our study is the conversion from reddening to gas mass. Deep Hi observations of the galaxies in this sample could constrain the amount of cold gas more directly, although the required integration times would be very large. The amount of cold gas can also be constrained through its effect on the star formation rate, which can be done by analyzing the spectra and spectral energy distributions of the galaxies (see Kaviraj \& van Dokkum 2008). Finally, it will be interesting to search for analogs of these systems at even lower redshifts.

We thank the referee for helpful comments. Support from NASA grant HST GO-10809.01-A is gratefully acknowledged.

limit (van Dokkum \& Franx 1995; Tomita et al. 2000).

\section{REFERENCES}

Bell, E.F., et al., 2004,ApJ, 608, 752.

Bell, E.F., Phleps, S., Somerville, R.S., Wolf, C., Borch, A., \& Meisenheimer, K., 2006, ApJ, 652, 270.

Cardelli, J.A., Clayton, G.C., Mathis, J.S., 1989, ApJ, 345, 245.

Croton, D.J., et al., 2006, MNRAS, 365, 11.

de Lucia, G., Springel, V., White, S.D.M., Croton, D., \& Kauffmann, G., 2006, MNRAS, 366, 499.

Donovan, J.L., Hibbard, J.E., \& van Gorkom, J.H., 2007, AJ, 134,1118

Escala, A., Larson, R. B., Coppi, P. S., \& Mardones, D., 2005, ApJ, 630, 152.

Faber, S.M., et al. 2007, ApJ, 665, 265.
Feldmann, R., Mayer, L., \& Carollo, C.,M., 2008, arXiv:0801.4764v1.

Gallazzi, A., Charlot, S., Brinchmann, J., White, S.D.M., Tremonti, C.A., 2005, MNRAS, 362, 41.

Hoffman, L., Loeb, A., 2007, MNRAS, 377, 957.

Kauffmann, G., et al. 2003, MNRAS, 341, 33

Kaviraj, S., van Dokkum, P.G., 2008, submitted to ApJ.

McIntosh, D.H., et al., 2007, astro-ph/0710.2157v2.

Rieke, G.H., \& Lebofsky, M.J., 1985, ApJ, 288, 618.

Sadler, E.M., \& Gerhard O.E., 1985, MNRAS, 214, 177.

Sérsic, L.L., 1968, Atlas de Galaxias Australes.

Tomita, A., Aoki, K., Watanabe, M., Takata, T., \& Ichikawa, S., 2000, AJ, 120, 123. 
Tran, K.H., van Dokkum, P.G., Franx, M., Illingworth, G.D., Kelson, D.D., \& Schreiber, N.M.F., 2005, AJ, 627, L25. van Dokkum, P.G., \& Franx, M., 1995, AJ, 110, 2027.

van Dokkum, P.G., Franx, M., Fabricant, D., Kelson, D.D., Illingworth, G.D., 1999, ApJ, 520, L95.

van Dokkum, P.G., 2001, PASP, 113, 1420. van Dokkum, P.G., 2005, AJ, 130, 2647.

White, M., Zheng, Z., Brown, M.J.I., Dey, A., and Jannuzi, B.T., 2007, ApJ, 655, 69.

Zheng, Z., Coil, A.L., and Zehavi, I., 2007, ApJ, 667, 760. 